\title{
A Compact UWB Indoor and Through-Wall Radar with Precise Ranging and Tracking
}

\author{
Yinan Yu, Jian Yang, Tomas McKelvey, and Borys Stoew \\ Department of Signals and Systems, Chalmers University of Technology, 41296 Gothenburg, Sweden \\ Correspondence should be addressed to Jian Yang, jian.yang@chalmers.se
}

Received 19 February 2012; Revised 8 April 2012; Accepted 14 April 2012

Academic Editor: Francesco Soldovieri

Copyright () 2012 Yinan Yu et al. This is an open access article distributed under the Creative Commons Attribution License, which permits unrestricted use, distribution, and reproduction in any medium, provided the original work is properly cited.

Ultrawideband (UWB) technology has many advantages compared to its narrowband counterpart in many applications. We present a new compact low-cost UWB radar for indoor and through-wall scenario. The focus of the paper is on the development of the signal processing algorithms for ranging and tracking, taking into account the particular properties of the UWB CMOS transceiver and the radiation characteristics of the antennas. Theoretical analysis for the algorithms and their evaluations by measurements are presented in the paper. The ranging resolution of this UWB radar has achieved 1-2 mm RMS accuracy for a moving target in indoor environment over a short range, and Kalman tracking algorithm functions well for the through-wall detection.

\section{Introduction}

Due to its quite unique properties, ultrawideband (UWB) technology finds many applications in different areas, such as UWB sensor network for precise ranging and geolocation, UWB radar and imaging systems with superior penetration and high resolution, and super sensitive UWB radio astronomy $[1-3]$.

From the radar perspective, UWB systems exhibit several distinct advantages over the narrowband counterparts [4, 5]: (1) higher ranging resolution with higher penetration ability through lossy materials simultaneously; (2) enhanced target recognition; (3) better penetration ability to passive interference (i.e., rain, fog, and clutter); (4) very low cost, particularly, the Novelda UWB radar system used in this paper.

We present a new compact and low-cost UWB radar system for ranging and tracking of moving objects in indoor and through-wall environments. The UWB radar consists of a commercially available Novelda R2A UWB CMOS chip transceiver [6] and the recently developed UWB $2-15-\mathrm{GHz}$ self-grounded-Bow-Tie (SGBT) antennas [7, 8]; see Figure 1. The particular properties of the Novelda transceiver and the radiation characteristics of the antenna have been taken into account for the development of the ranging and tracking algorithms.

The ranging resolution of this UWB radar by applying the algorithm has achieved an accuracy of 1-2 mm RMS error for a moving target in indoor environments over a short range, and the Kalman filter tracking algorithm functions well for a through-wall detection.

In this paper, the system is briefed in Section 2. The selfgrounded Bow-Tie antenna is described in Section 2.2. The system signal is modeled in Section 3. Algorithms of adaptive clutter mapping are presented in Section 4 . Afterwards, the algorithms for ranging are described in detail in Section 5. An important issue, calibration of the system, is discussed in Section 6. A Kalman tracking algorithm based on the pulse-spectrum ranging is briefed in Section 7. Then, the evaluation of the algorithms by measurements is illustrated in Section 8.

\section{The Radar System}

2.1. Overview of the System. The UWB radar system consists of two SGBT antennas and one Novelda transceiver; see Figure 1. Pulses are transmitted via one antenna 


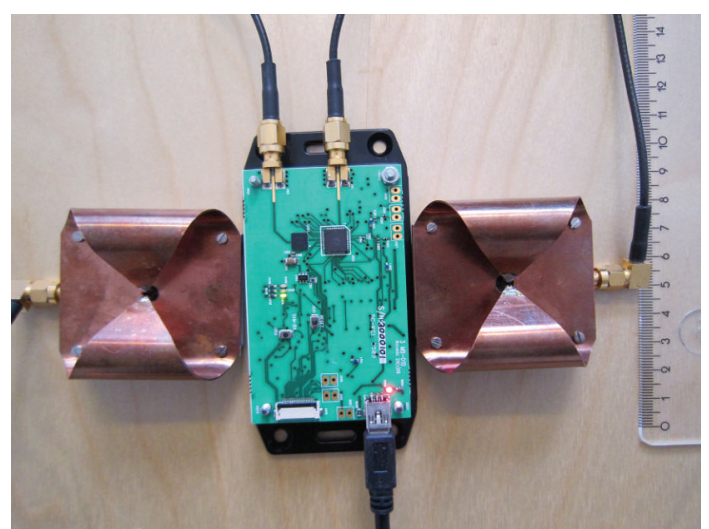

FIGURE 1: The compact UWB radar system for indoor and throughwall ranging and tracking.

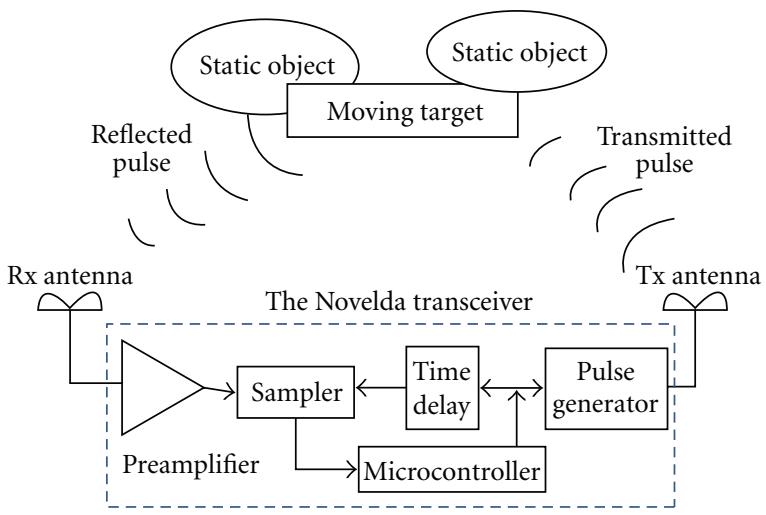

FIGURE 2: Presentation of the operating principle of the compact UWB radar system.

(Tx antenna), reflected by targets, and then received by the second antenna (Rx antenna), as depicted in Figure 2.

For memory efficiency, each measurement by the Novelda transceiver is taken within a fixed time frame (referred to as the measurement frame, or simply the frame), with an offset time delay predefined by user. Each measurement has 128 samples. The time interval (or step) between two consecutive samples is $27.8 \mathrm{ps}$, corresponding to the sample resolution of $4.167 \mathrm{~mm}$ in the wave propagation direction. The duration of one frame is then $127 \times 27.8 \mathrm{ps}=3.53 \mathrm{~ns}$ in time and $529 \mathrm{~mm}$ in space accordingly.

The received signals can be amplified by user-defined values in the transceiver. The signal sample data are then sent to the microcontroller for data processing.

2.2. The Self-Grounded Bow-Tie Antenna. The self-grounded Bow-Tie (SGBT) antenna, shown in Figure 1, is a new type of UWB antenna, developed recently at Chalmers University of Technology $[7,8]$. Several other types of UWB antennas have been also developed at Chalmers for different applications [9-12].

The SGBT antenna is chosen for this UWB radar system due to the following characteristics: (1) very good time response; (2) compact size and low profile; (3) UWB constant directional radiation beam; (4) good UWB reflection coefficient; (5) better penetration ability than other UWB antennas $[8,13]$. Antipodal Vivaldi antennas were used in the previous work [14]. With the use of the SGBT antenna, the performance of the UWB radar system has been improved, due to its good time response and UWB constant directional radiation beam.

The size of the SGBT antenna is $54 \times 58 \times 24 \mathrm{~mm}^{3}$, with the operating frequency band covering $2-15 \mathrm{GHz}$. The details of the measured and simulated performance of the antenna can be found in $[7,8]$.

The comparison between the self-grounded Bow-Tie antenna and a Vivaldi antenna with a size of $135 \times 145 \times$ $2 \mathrm{~mm}^{3}$ on the penetration ability through a concrete wall was presented in [8], which indicated that the system using the self-grounded Bow-Tie antenna has $6 \mathrm{~dB}$ higher signal level that is using the Vivaldi antenna. The antipodal Vivaldi antennas used in [14] have even lower penetration ability than the aforementioned Vivaldi antenna.

The measured time-domain response of the UWB radar system is shown in Figure 3, when the two antennas are separated by a distance of $250 \mathrm{~mm}$ in the face-to-face configuration in an anechoic chamber. From the figure, two time-response measures, the width of the pulse full-width at half-maximum (FWHM) $\tau_{\mathrm{FWHM}}$ and the duration of the ringing $\tau_{r=0.22}$, can be obtained as $\tau_{\mathrm{FWHM}}=134 \mathrm{ps}$ and $\tau_{r=0.22}=260 \mathrm{ps}$, which states a fast time pulse response of the radar system. Please refer to [15] for the definitions of $\tau_{\mathrm{FWHM}}$ and $\tau_{r=0.22}$.

Note that in the antenna setup of the system, it is required that the target should be a certain distance away from the antennas for this radar system to have an accurate ranging, because then the target pulse can be distinguished clearly from the direct-coupling pulse between the antennas. Since the pulse used in this work has a width of about $0.5 \mathrm{~ns}$ (Figure 3), which corresponds to $150 \mathrm{~mm}$, the distance between the target and the antennas should be larger than $150 \mathrm{~mm}$. With enough margin, it is set in this work that the minimum distance between the target and the antennas should be larger than both the distance between the two antennas and $200 \mathrm{~mm}$ for an accurate ranging result.

\section{Signal Model}

Figure 4 shows the signal model used in this work, where the received signal $\mathbf{r}$ consists of the clutter $\mathbf{r}_{e}$ (unwanted reflected signal from a static indoor environment, such as a wall), the target signal $\mathbf{r}_{t}$ that is reflected from moving targets, and the noise e. Note that the definitions of the clutter and the target can be different in different applications. The noise $\mathbf{e}$ is defined as the channel noise, modeled by a zero-mean random variable. Here, we assume that there is no jamming, namely, no other sources of signals within the field of the radar system.

In the Novelda transceiver, each measurement consists of 128 samples. The received signal $\mathbf{r}$ and its subcomponents $\mathbf{r}_{e}$, 


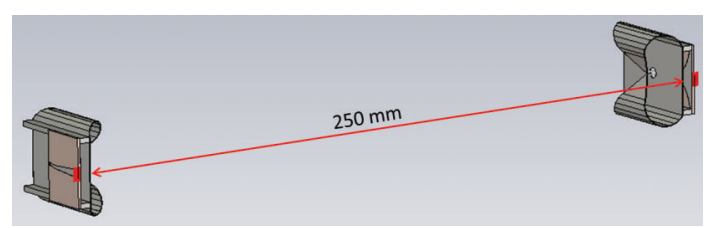

(a)

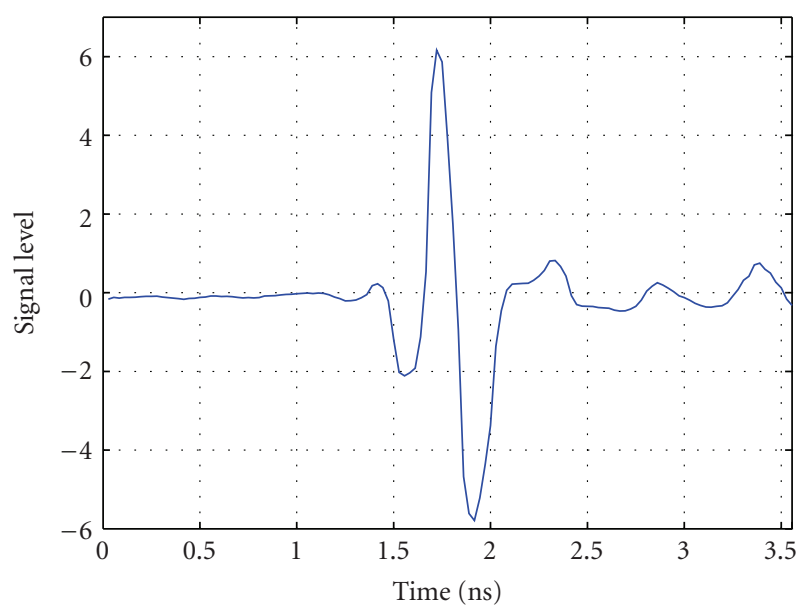

(b)

FIGURE 3: Measured time-domain impulse response of the UWB radar system when the two antennas are separated by $250 \mathrm{~mm}$ in the face-to-face configuration.

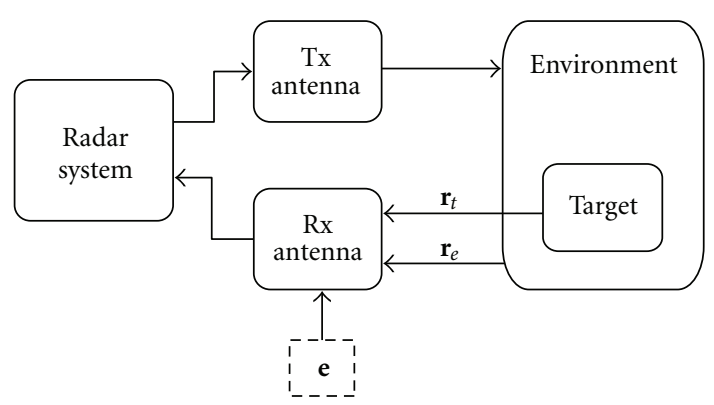

Figure 4: Signal system model, where the received signal $\mathbf{r}$ is constructed of three additive parts: the clutter $\mathbf{r}_{e}$, the target signal $\mathbf{r}_{t}$, and the noise e.

$\mathbf{r}_{t}$, and $\mathbf{e}$ are therefore expressed by 128-dimensional vectors as

$$
\mathbf{r}=\mathbf{r}_{e}+\mathbf{r}_{t}+\mathbf{e},
$$

where

$$
\mathbf{r}=[r(1), r(2), \ldots, r(128)]^{T},
$$

with $T$ transposition, and the same for $\mathbf{r}_{e}, \mathbf{r}_{t}$, and $\mathbf{e}$.

Let vectors $\mathbf{r}^{i-(n-1)}, \mathbf{r}^{i-(n-2)}, \ldots, \mathbf{r}^{i-1}, \mathbf{r}^{i}$ denote the measurements at time $t_{i-(n-1)}, t_{i-(n-2)}, \ldots, t_{i-1}, t_{i}$, and denote a measurement matrix $\mathbf{X}^{i}$ at time $t_{i}$ by the present and past $n$ measurements as

$$
\mathbf{X}^{i}=\left[\mathbf{r}^{i-(n-1)}, \mathbf{r}^{i-(n-2)}, \ldots, \mathbf{r}^{i-1}, \mathbf{r}^{i}\right]
$$

From (1), the measurement matrix $\mathbf{X}^{i}$ can be written as

$$
\mathbf{X}^{i}=\mathbf{X}_{e}^{i}+\mathbf{X}_{t}^{i}+\mathbf{E}^{i}
$$

where $\mathbf{X}_{e}^{i}, \mathbf{X}_{t}^{i}$, and $\mathbf{E}^{i}$ are referred to as the clutter matrix, the target matrix, and the noise matrix, respectively.

\section{Adaptive Clutter Mapping}

The Novelda transceiver has a particular property: the received signal at each measurement may be rescaled and shifted by a bias. This means that even in a static environment, the clutter map may change with the measurement. Without losing generality, each clutter measurement $\mathbf{r}_{e}^{i}$ can be modeled by a rescaling factor $\alpha^{i}$ and a shifted bias $c^{i}$ as

$$
\mathbf{r}_{e}^{i}=\alpha^{i} \mathbf{r}_{c}+\mathbf{c}^{i}
$$

where $\mathbf{r}_{c}$ represents the clutter vector, bias vector $\mathbf{c}^{i}=\left[\begin{array}{c}1 \\ \vdots \\ i\end{array}\right] c^{i}$, and $c^{i}$ is a constant. Therefore,

$$
\begin{aligned}
\mathbf{X}_{e}^{i} & =\left[\alpha^{i-(n-1)} \mathbf{r}_{c}+\mathbf{c}^{i-(n-1)}, \ldots, \alpha^{i} \mathbf{r}_{c}+\mathbf{c}^{i}\right] \\
& =\left[\alpha^{i-(n-1)} \mathbf{r}_{c}, \ldots, \alpha^{i} \mathbf{r}_{c}\right]+\left[\mathbf{c}^{i-(n-1)}, \ldots, \mathbf{c}^{i}\right] \\
& =\mathbf{R}_{c}^{i}+\mathbf{C}^{i},
\end{aligned}
$$

where

$$
\begin{aligned}
\mathbf{R}_{c}^{i} & =\left[\alpha^{i-(n-1)}, \ldots, \alpha^{i}\right] \mathbf{r}_{c}, \\
\mathbf{C}^{i} & =\left[\mathbf{c}^{i-(n-1)}, \ldots, \mathbf{c}^{i}\right] .
\end{aligned}
$$

The maximum rank of the clutter matrix $\mathbf{X}_{e}^{i}$ is 2 by the assumption that there are only two independent vectors $\left[\alpha^{i-(n-1)}, \ldots, \alpha^{i}\right]$ and $\left[\mathbf{c}^{i-(n-1)}, \ldots, \mathbf{c}^{i}\right]$.

Due to the property of (6), the method of singular value decomposition (SVD) [16] is used for the clutter mapping, referred to as the SVD clutter mapping. 
Suppose that $\mathbf{X}^{i}$ is an $m \times n$ matrix with a rank of $L$. Then, it can be factorized as

$$
\mathbf{X}^{i}=\mathbf{U} \Sigma^{i} \mathbf{V}^{H}=\mathbf{U}\left(\begin{array}{cccccc}
\sigma_{1}^{i} & 0 & \cdots & \cdots & \cdots & 0 \\
\vdots & \ddots & 0 & \cdots & \cdots & 0 \\
0 & \cdots & \ddots & 0 & \cdots & 0 \\
0 & 0 & \cdots & \sigma_{L}^{i} & \cdots & 0 \\
0 & \cdots & \cdots & \cdots & 0 & 0 \\
\vdots & \vdots & \vdots & \vdots & \vdots & \vdots \\
0 & 0 & \cdots & \cdots & \cdots & 0
\end{array}\right) \mathbf{V}^{H},
$$

where $\mathbf{U}$ is an $m \times m$ unitary matrix, $\Sigma^{i}$ is an $m \times n$ matrix with diagonal entries of nonnegative-real numbers, and $\mathbf{V}$ is an $n \times n$ unitary matrix. The nonzero entries $\sigma_{l}^{i}, l=1, \ldots, L$, the singular values of matrix $\mathbf{X}^{i}$, are sorted in the descending order. Thus, $\mathbf{X}^{i}$ can be expressed by a summation of $L$ onerank matrices:

$$
\mathbf{X}^{i}=\sum_{l=1}^{L} \mathbf{u}_{l} \sigma_{l}^{i} \mathbf{v}_{l}^{H}
$$

where $\mathbf{u}_{l}, \mathbf{v}_{l}^{H}$, and $\sigma_{l}^{i}$ are the left eigenvector, the right eigenvector, and the singular value of $\mathbf{X}^{i}$, respectively.

According to (6), the two strongest singular values represent the clutter map, that is,

$$
\widehat{\mathbf{r}}_{e}^{i}=\sum_{l=1}^{2} \mathbf{u}_{l} \sigma_{l}^{i}\left(\mathbf{v}_{l}^{i}\right)^{H}
$$

where $\hat{\mathbf{r}}_{e}^{i}$ is defined as in (5) for the $i$ th measurement $\mathbf{r}^{i}$. Therefore, the clutter map can be removed from the measurement $\mathbf{r}^{i}$, and the reflection of the target can be estimated accordingly:

$$
\widehat{\mathbf{r}}_{t}^{i}=\mathbf{r}^{i}-\widehat{\mathbf{r}}_{e}^{i} .
$$

An example of the clutter mapping is shown in Figure 5: 100 consecutive measurements $(n=100)$ of a moving metal plate $\left(225 \times 500 \mathrm{~mm}^{2}\right)$. The overlapping part of all measurements represents the clutter map $\widehat{\mathbf{r}}_{e}^{i}$, and the pulses different from the clutter map indicate the echoes from the target $\mathbf{r}_{t}^{i}$. It is observed from the figure that although the clutter is static, the clutter map has a certain variation (a thick belt of the overlapped curve). This is due to the rescaling factor $\alpha^{i}$ and the shifted bias $c^{i}$ of the Novelda transceiver, as described in (5).

The choice of the number of measurements $n$ in the measurement matrix $\mathbf{X}^{i}$ for the clutter mapping depends on the applications. If $n$ is large, the clutter map is more related to the static environment with a higher computational complexity. If $n$ is small, any object that appears to be static for a short time or moves slowly will be considered as a part of the clutter, with a shorter computation time.

As a reference, the direct mean method is also used for the clutter mapping (referred to as the direct-mean method) for each measurement, which is defined as

$$
\hat{\mathbf{r}}_{e}^{i}=\frac{1}{n} \sum_{k=i-(n-1)}^{i} \mathbf{r}^{k}
$$

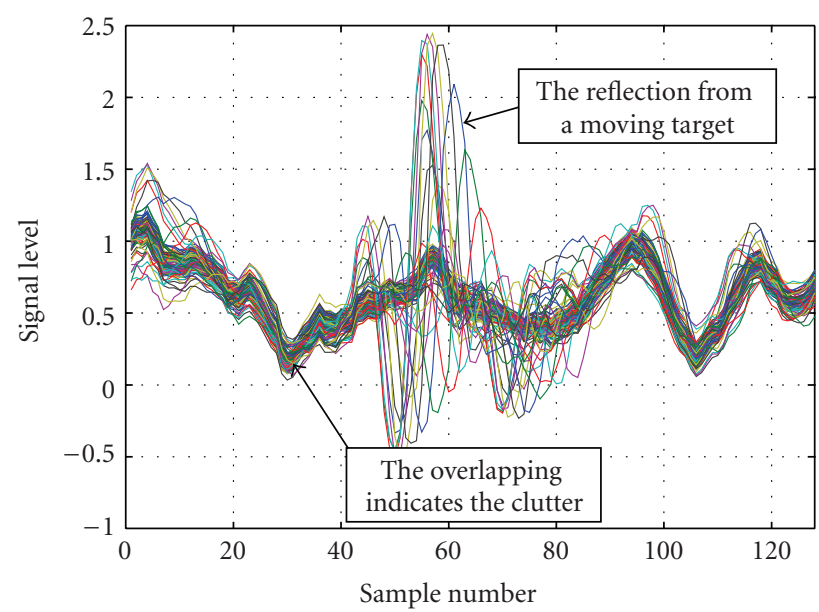

FIGURE 5: 100 consecutive measurements of a moving metal plate $\left(225 \times 500 \mathrm{~mm}^{2}\right)$ in the presence of clutter. The overlapping part represents the clutter map $\widehat{\mathbf{r}}_{e}^{i}$, and the pulses different from the overlapping part indicate the target signals $\mathbf{r}_{t}^{i}$.

that is, in the direct-mean method, the clutter map $\widehat{\mathbf{r}}_{e}^{i}$ is obtained by averaging the measurements $\mathbf{r}^{k}, k=i-n+1, i-$ $n+2, \ldots, i$, over the $n$ measurements. It is obvious that this method assumes that the rescaling and the bias shifting and the reflection from a moving target are zero-mean random variables, which is not always true in reality.

\section{Ranging}

Three ranging techniques have been investigated. The first two are sample based, which means that the ranging is obtained by finding the sample index of the pulse front in the received target signal. The last one is a fractional-sample method, in order to get a higher ranging resolution.

5.1. Pulse Signature Matching. The received pulse signature $\mathbf{r}_{s}$ is defined first in this method.

In order to maintain the robustness over a range of objects, the pulse signature is obtained by (1) averaging target pulse signals over 20 different targets, measured in anechoic chamber; (2) using a rectangular window (referred to as the pulse signature window) to select the main lobes of the averaged pulse signal $\mathbf{r}_{\text {avg }}$ as the pulse signature.

Referring to Figure 6, we define

$$
r_{s}(m)= \begin{cases}0, & m<0 \\ r_{\mathrm{avg}}\left(n_{\mathrm{st}}+m\right), & 0 \leq m \leq N_{w} \\ 0, & m>N_{w}\end{cases}
$$

where $n_{\mathrm{st}}$ and $N_{w}$ are the starting sample index and the sample width of the pulse signature window in the averaged signal, respectively. 


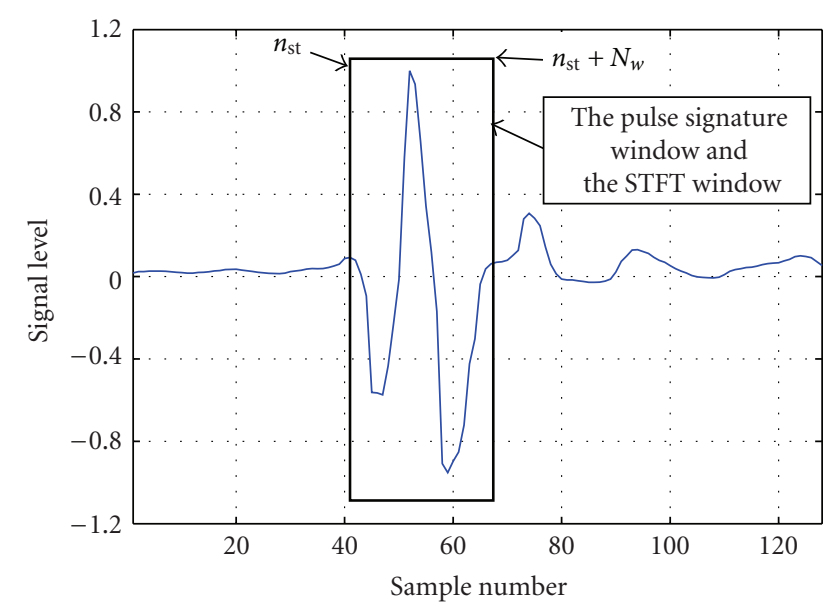

Figure 6: The pulse signature is defined by the main lobes (inside the pulse signature window) of the averaged target signals over 20 different targets measured in anechoic chamber. The same window sliding through the target signal sequence is used for STFT in pulsespectrum signature matching.

Then, the cross-correlation $X_{\text {corr }}(k)$ between the target signal $\mathbf{r}_{t}$ and the pulse signature $\mathbf{r}_{s}$ with different time delay $k$ is calculated by

$$
X_{\text {corr }}(k)=\left|\sum_{m=1}^{128} r_{t}(m) r_{s}(m-k)\right|, \quad 1 \leq k \leq 128-N_{w} .
$$

The peak value of $X_{\text {corr }}(k)$ appears obviously at such a time delay $k$ that the pulse front of the pulse signature coincides with the pulse front of the target signal. Therefore, the sample index of the target pulse front $\hat{j}$, referred to as the target index, is estimated by

$$
\hat{j}=\underset{k \in\left(1,128-N_{w}\right)}{\arg \max } X_{\text {corr }}(k) .
$$

In a traditional radar problem, this classic approach is referred to as matched filter $[17,18]$. It has its advantage of simplicity. However, the similarity between the target signal pulse and the pulse signature plays an important role for the accuracy of this method. In far-field applications, the distance between the radar system and the target is much larger than that of the pulse duration. Namely, the time it takes for the reflected signal coming back to the receiver is much longer than the signal duration itself, and thus the changing of the pulse shape can be ignored in this case [19]. However, in short-range indoor applications, the shape difference between the target signal and the pulse signature will cause measurement errors. In addition, multiple reflections between the target and the clutter may happen and lead to complicated scenario. For example, the ringing part can be very similar to the main portion of the pulse, in both the shape and the amplitude. A big ranging error may arise in such cases.
5.2. Pulse-Spectrum Signature Matching. The UWB signal spans a large spectrum, and it has been observed in this work that the spectrum of the Novelda transceiver together with the SGBT antennas within a main bandwidth (such as 2$4 \mathrm{GHz}$ in this work) does not severely vary with different scenarios. In other words, the changes of the spectrum with respect to different targets and environments appear mainly in a few frequencies, while the change of the pulse shape in time domain can be significant. It is then natural to take advantage of this spectrum-stable property, instead of using only time-domain analysis. A joint time-frequency domain ranging method, the pulse-spectrum signature matching, is therefore introduced, as follows.

(1) The Short-Time Fourier Transform (STFT) [20, 21], is applied in the first step to the target signal as

$$
R_{t}(k, \omega)=\sum_{m=0}^{N_{w}-1} r_{t}(m+k) W(m) e^{-j \omega m},
$$

where $r_{t}(m)$ is the sample of the target signal, $W(m)$ is the sample of a window function $W$, angular frequency $\omega=$ $0,2 \pi\left(1 / N_{w}\right), \ldots, 2 \pi\left(\left(N_{w}-1\right) / N_{w}\right)$, and $j$, imaginary unit. In this work, a rectangular window function (referred to as the STFT window), with the same width of $N_{w}$ as that of the pulse signature window, is used; see Figure 6.

Within each window, the short-time Fourier transform in (16) can be rewritten by the multiplication of the target signal $\mathbf{r}_{t}$ with an $N_{w}$-point discrete Fourier transform (DFT) matrix $\mathbf{W}[22,23]$ defined as

$$
\mathbf{W}=\frac{1}{\sqrt{N_{w}}}\left[\begin{array}{cccc}
1 & 1 & \cdots & 1 \\
1 & e^{-j\left(2 \pi / N_{w}\right)} & \cdots & e^{-j\left(2\left(N_{w}-1\right) \pi / N_{w}\right)} \\
1 & e^{-j\left(4 \pi / N_{w}\right)} & \cdots & e^{-j\left(4\left(N_{w}-1\right) \pi / N_{w}\right)} \\
1 & e^{-j\left(6 \pi / N_{w}\right)} & \cdots & e^{-j\left(6\left(N_{w}-1\right) \pi / N_{w}\right)} \\
\vdots & \vdots & \vdots & \\
1 & e^{-j\left(2\left(N_{w}-1\right) \pi / N_{w}\right)} & \cdots & e^{-j\left(2\left(N_{w}-1\right)^{2} \pi / N_{w}\right)}
\end{array}\right] .
$$

In general, we can define the STFT window function by

$$
\mathbf{W}^{\prime}=\operatorname{diag}(\boldsymbol{\beta}) \mathbf{W},
$$

where $\boldsymbol{\beta}=\left(\beta_{1}, \beta_{2}, \ldots, \beta_{N_{w}}\right)$ is a coefficient vector whose entry gives a weight to each frequency component, which can also be referred to as the spectrum signature window, shown in Figure 7. In this work, we set $\beta_{i}=1$ for all the frequencies in the band of $2-4 \mathrm{GHz}$, and $\beta_{i}=0$ for the frequencies out of the band.

(2) The Spectrum Signature is defined in the second step. Applying the same STFT window as the pulse signature window (the same width and sample index location) to the pulse signature $\mathbf{r}_{s}$, the spectrum of the pulse signature can be obtained by $\mathbf{W}^{\prime} \mathbf{r}_{s}$, shown in Figure 7 . Here by using $\mathbf{W}^{\prime}$, we use only the main part of the whole spectrum inside the spectrum signature window as the spectrum signature, for simplicity and robustness of the algorithm, such as the band from 2 to $4 \mathrm{GHz}$. 


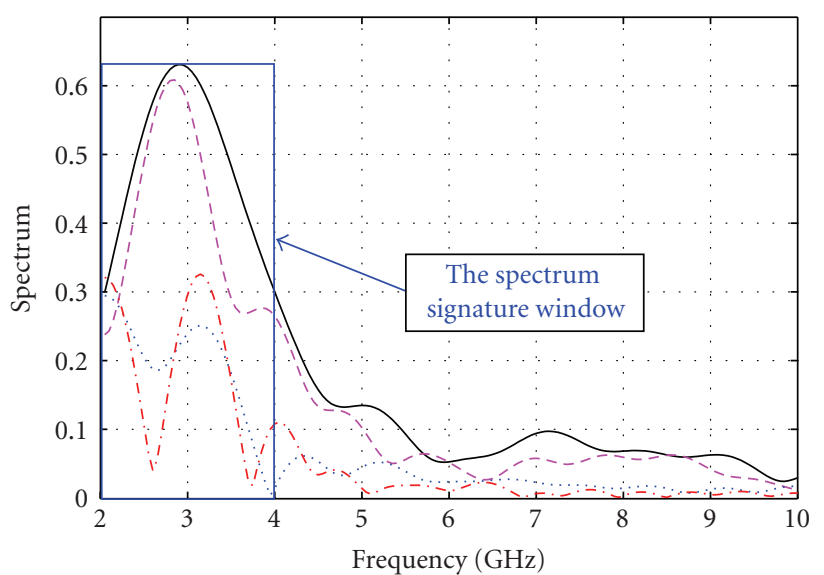

$$
\begin{aligned}
& \text { - Spectrum signature } \\
& -- \text { Spectrum when the main lobes of } \\
& \text { signal are inside STFT window } \\
& \text {.... Spectrum when STFT window is } \\
& \text { at another place } 1 \\
& \text {-.- Spectrum when STFT window is } \\
& \text { at another place } 2
\end{aligned}
$$

FIGURE 7: The different spectrums when the STFT window slides through the target signal. The solid black line presents the spectrum signature.

(3) The Spectrum Signature Matching is then introduced. The cross-correlation $X_{\text {corr } f}(k)$ is calculated, similar to the pulse signature matching method, by

$$
X_{\mathrm{corr}_{f}}(k)=\left|\left[\mathbf{W}^{\prime} \mathbf{r}_{t}\left(k: k+N_{w}\right)\right]^{*} \cdot\left(\mathbf{W}^{\prime} \mathbf{r}_{s}\right)\right|
$$

with $k \in\left(1,128-N_{w}\right)$, and the superscript $*$ conjugation. A clearly high correlation can be found between the spectrum signature and the spectrum of the target signal when the STFT window is located at such a position that the main lobes of the target signal are inside it, while for the other positions, the correlation is low. The target index $\hat{j}$ can therefore be estimated by

$$
\hat{j}=\underset{k \in\left(1,128-N_{w}\right)}{\arg \max } X_{\operatorname{corr}_{f}}(k) .
$$

(4) The Pulse and the Spectrum Signature Matchings are combined in the final step, in order to achieve a more accurate and robust ranging algorithm. A linear combination of the pulse and the spectrum signature matchings is applied as

$$
X_{\text {com_corr }}(k)=(1-\gamma) X_{\text {corr }}^{\prime}(k)+\gamma X_{\text {corr }_{f}}^{\prime}(k),
$$

with $k \in\left(1,128-N_{w}\right)$, where the normalized crosscorrelations in time and frequency domains are defined by

$$
\begin{aligned}
X_{\text {corr }}^{\prime}(k) & =\frac{X_{\text {corr }}(k)}{\max \left(X_{\text {corr }}(k)\right)}, \\
X_{\text {corr }_{f}}^{\prime}(k) & =\frac{X_{\text {corr }_{f}}(k)}{\max \left(X_{\text {corr }_{f}}(k)\right)},
\end{aligned}
$$

where $\max (x)$ is the maximum value of the vector $x$.

The final estimated target index $\hat{j}$ (therefore the ranging) is found by

$$
\hat{j}=\underset{k \in\left(1,128-N_{w}\right)}{\arg \max } X_{\text {com_corr }}(k) .
$$

The empirical constant $\gamma=0.7$ is determined by crossvalidation.

5.3. Subsample Delay Estimation. The above-discussed ranging approaches are sample based. The best achievable ranging resolution is therefore half the sample resolution: $4.167 / 2=2.08 \mathrm{~mm}$ (refer to Section 2). In order to obtain a better ranging resolution, the fractional sample shift has to be determined. An estimation of subsample delay based on the Fourier transform is introduced as follows.

Suppose that the target sample index $\hat{j}$ has been obtained by the pulse-spectrum method, and actually the target pulse front of $r_{t}$ is located at sample $\hat{j}+\hat{j_{\tau}}$, where $\left|\hat{j}_{\tau}\right|<1$ is a fractional sample delay around $\hat{j}$. Then, we locate the pulse signature $r_{s}$ at sample $\hat{j}$ and let $r_{t_{\text {main }}}=r_{t}\left(\hat{j}: \hat{j}+N_{w}-1\right)$ be the main portion of the received pulse. Since $r_{t_{\operatorname{man}}}$ and $r_{s}$ have a slight difference in both shape and phase, applying the Fourier transform, we get the following relation for the transformed signals $R_{t}(\omega)$ and $R_{s}(\omega)$ [24]:

$$
\frac{R_{t}(\omega)}{R_{s}(\omega)}=C(\omega) e^{-j \omega \tau},
$$

where $\tau$ is the time delay between $r_{t_{\text {main }}}$ and $r_{s}$, and $C(\omega)$ results from the shape difference.

However, since the main portion of the signal does not change significantly and therefore the pulse signature $r_{s}$ is assumed to be identical in shape compared to $r_{t_{\text {main }}}$, namely, we have

$$
r_{t_{\text {main }}}(t) \approx r_{s}(t-\tau)
$$

and thus,

$$
\frac{R_{t}(\omega)}{R_{s}(\omega)} \approx e^{-j \omega \tau}
$$

By retrieving the angle, we have

$$
\angle \frac{R_{t}(\omega)}{R_{s}(\omega)} \approx-\omega \tau \text {. }
$$

The above expression is valid for all frequency points within the bandwidth defined by the spectrum signature window, that is,

$$
\omega \in\left[\omega_{l}, \omega_{l+1}, \ldots, \omega_{h}\right]=2 \pi\left[f_{l}, f_{l+1}, \ldots, f_{h}\right],
$$

where $f_{l}$ and $f_{h}$ are the frequency points of the low and the high ends of the bandwidth, respectively. For example, $f_{l}=2 \mathrm{GHz}$ and $f_{h}=4 \mathrm{GHz}$ in this work, shown in Figure 7. Therefore, the slight time shift $\tau$ can be retrieved from the above linear relation by least squares estimation. 


\section{Adaptive Calibration by Direct Coupling}

Adaptive calibration is a necessity for this UWB radar system due to the following. (1) The heating of the radar device due to a long time continuous usage may cause different time delays of the transmitted pulses. (2) The cables connecting the transceiver to the antennas may have different conditions, such as the length and the bending shape, which also cause different time delays. An adaptive calibration by using the direct coupling between the two antennas is therefore introduced.

The estimated distance $d_{t}^{i}$ between the target and the antenna center at the $i$ th measurement can be expressed by

$$
d_{t}^{i}=\frac{\Delta_{\tau}\left(\hat{j}_{t}^{i}-\hat{j}_{a t}^{i}\right)}{2}
$$

where $\Delta_{\tau}$ is the ranging resolution between two consecutive samples, $\hat{j}_{t}^{i}$ is the target index, and $\hat{j}_{a t}^{i}$ is the antenna-center pulse sample index (the pulse sample index when the pulse front is passing the Tx antenna center). Here, $\Delta_{\tau}$ and $\hat{j}_{t}^{i}$ are known, but $\hat{j}_{a t}^{i}$ is unknown.

Now, we utilize the direct coupling between the two antennas to eliminate the unknown parameter $\hat{j}_{a t}^{i}$.

Assume that the distance between the two antennas $d_{\text {ant }}$ is smaller than that between the antennas and the target. Since $d_{\text {ant }}$ is fixed and known, and the direct coupling is the first received pulse, $d_{\text {ant }}$ can be expressed by

$$
d_{\mathrm{ant}}=\Delta_{\tau}\left(\hat{j}_{a r}^{i}-\hat{j}_{a t}^{i}\right),
$$

where $\hat{j}_{a r}^{i}$ is the sample index of the first received pulse determined by using the ranging methods discussed in the previous section. Therefore, from (29) and (30), we have

$$
d_{t}^{i}=\frac{\Delta_{\tau}\left(\hat{j}_{t}^{i}-\hat{j}_{a r}^{i}\right)+d_{\mathrm{ant}}}{2} .
$$

\section{Tracking}

A Kalman filter [25] tracking algorithm is applied in this work, and the pulse-spectrum signature matching with the subsample delay estimation is used as the measurements in the tracking algorithm.

7.1. One-Dimensional Tracking. The state $\mathbf{x}_{k}$ is constructed of the position $p_{k}$ and the velocity $\dot{p}_{k}$ as

$$
\mathbf{x}_{k}=\left[p_{k}, \dot{p}_{k}\right]^{T} .
$$

The measurement $\mathbf{z}_{k}$ is obtained by using the ranging method presented in Section 5 . Then the state $\mathbf{x}_{k}$ is adaptively corrected in real time by the Kalman filter update.

7.2. Two-Dimensional Tracking. For two-dimensional space, a pair of the UWB radar systems are set up orthogonally,

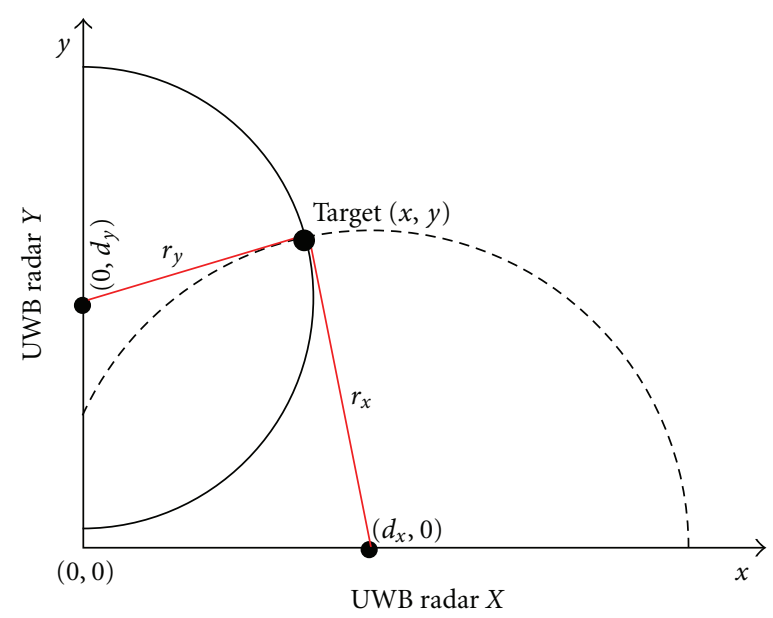

Figure 8: Setup for 2-dimensional tracking.

as shown in Figure 8, for the two-dimensional tracking. The coordinate $(x, y)$ of the target position can be calculated by

$$
\begin{aligned}
& x=d_{x}-r_{x} \cos \left(\theta_{x}\right), \\
& y=d_{y}-r_{y} \cos \left(\theta_{y}\right),
\end{aligned}
$$

where $r_{x}$ and $r_{y}$ are the ranging values measured by UWB radars $X$ and $Y$, with their positions at $\left(d_{x}, 0\right)$ and $\left(0, d_{y}\right)$, respectively, and

$$
\begin{gathered}
\theta_{x}=\arccos \left(\frac{d^{2}+r_{x}^{2}-r_{y}^{2}}{2 d r_{x}}\right)+\arccos \left(\frac{d_{x}}{d}\right), \\
\theta_{y}=\arccos \left(\frac{d^{2}+r_{y}^{2}-r_{x}^{2}}{2 d r_{y}}\right)+\arccos \left(\frac{d_{y}}{d}\right), \\
d^{2}=d_{x}^{2}+d_{y}^{2} .
\end{gathered}
$$

By extending the Kalman filter to the two-dimensional case, the state and the observation vectors are defined as

$$
\begin{aligned}
& \mathbf{x}_{k}^{\prime}=\left[x_{k}, \dot{x}_{k}, y_{k}, \dot{y}_{k}\right]^{T}, \\
& \mathbf{z}_{k}^{\prime}=\left[z_{x k}, z_{y k}\right]^{T},
\end{aligned}
$$

where $z_{x k}$ and $z_{y k}$ are the observation values of $x$ and $y$ coordinates, respectively, calculated based on the measurements $r_{x}$ and $r_{y}$ by (33).

More details about Kalman filter equations and update can be found in $[25,26]$.

\section{Measurement Evaluations}

All algorithms developed for clutter mapping, ranging, and tracking are evaluated by the measurements.

8.1. Clutter Mapping. Both the SVD and the direct-mean methods have been evaluated in an indoor environment with and without moving targets.

Without moving targets, the measurement consists of only the clutter and noise: $\mathbf{r}_{0}^{i}=\mathbf{r}_{e}^{i}+\mathbf{e}^{i}$, and we assume that 


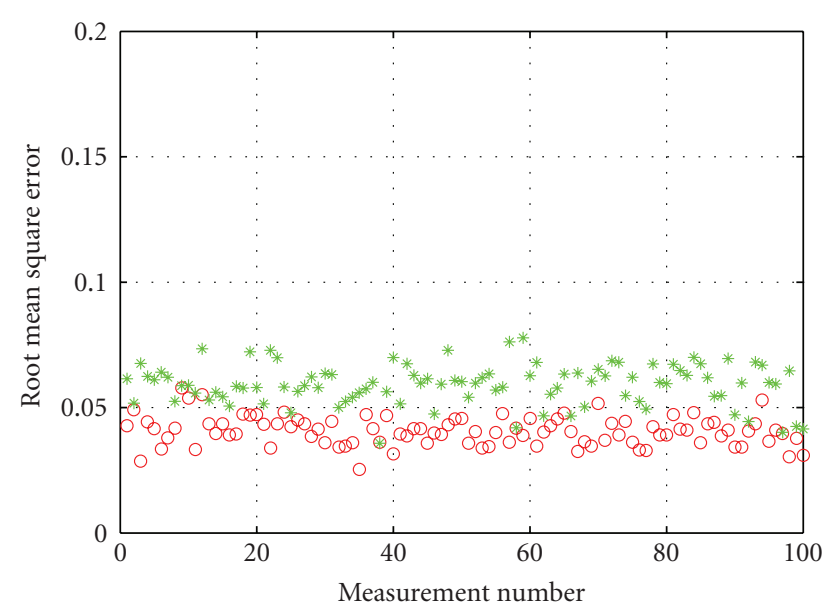

SVD clutter mapping

* Direct-mean clutter mapping

FIGURE 9: RMSE values for evaluation of the SVD and the directmean method without moving target.

the noise level is much lower than that of the clutter. Keep in mind that in spite of the static clutter, the received signal $\mathbf{r}_{0}^{i}$ varies a bit due to the rescaling factor and the shifted bias.

The evaluation is carried out in terms of the root-meansquare error (RMSE) between the clutter map $\widehat{\mathbf{r}}_{e}^{i}$ (Section 4) and the real measurement $\mathbf{r}_{0}^{i}$, which can be expressed by

$$
\operatorname{RMSE}_{\text {Clutter }}=\sqrt{\frac{1}{n} \sum_{i=1}^{n}\left|\widehat{\mathbf{r}}_{e}^{i}-\mathbf{r}_{0}^{i}\right|^{2}}
$$

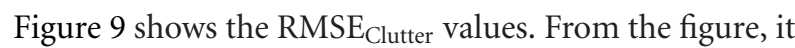
can be observed that the SVD clutter mapping gives smaller RMSE values than the direct-mean method does, stating the superiority of the SVD clutter mapping.

With the presence of moving targets in the clutter, the evaluation becomes complicated since both the clutter map and the target signal vary. An off-line emulating method is therefore introduced for the evaluation of this case as follows.

A moving target is measured in the anechoic chamber at our lab as $\mathbf{r}_{t}^{i}$, where it is assumed no clutter. Then, the measured data $\mathbf{r}_{t}^{i}$ is added to the previous measurements of the clutter without moving targets $\mathbf{r}_{0}^{i}=\mathbf{r}_{e}^{i}+\mathbf{e}^{i}$ to emulate the scenario of the target in the clutter by

$$
\mathbf{r}^{i}=\mathbf{r}_{t}^{i}+\mathbf{r}_{e}^{i}+\mathbf{e}^{i}
$$

The emulated measurements are shown in Figure 10, where $n=100$ in the measurement matrix $\mathbf{X}$. The RMSE of the clutter map $\widehat{\mathbf{r}}_{e}^{i}$ (by both the SVD and direct-mean clutter mappings) with respect to the clutter $\mathbf{r}_{0}^{i}$ is defined in the same way as (36). The data are shown in Figure 11, illustrating again the superiority of the SVD clutter mapping.

8.2. Ranging. The off-line emulating method is used for the evaluation of our ranging algorithms, since we do not have any other means to obtain the accurate ranging values of a

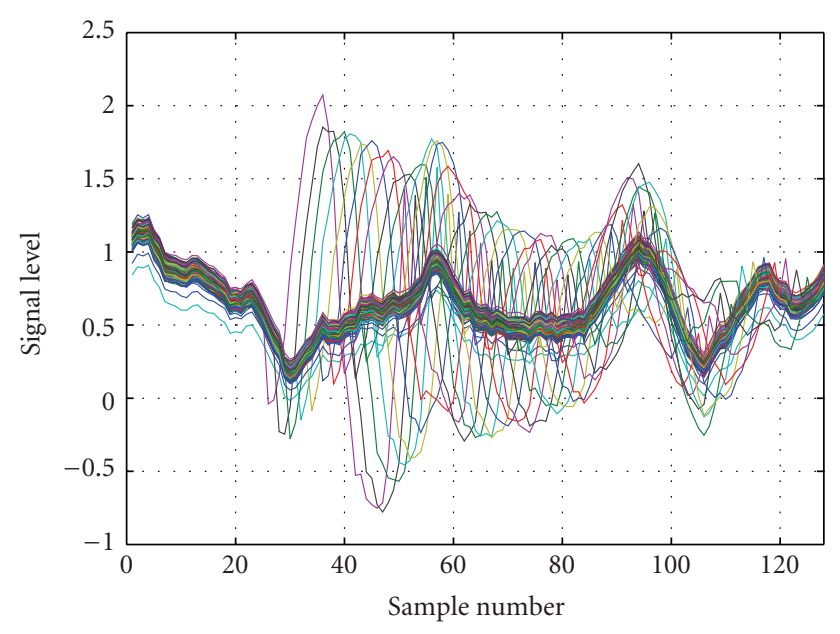

FIGURE 10: Emulated measurement matrix by adding the measurements of a moving target in anechoic chamber to the measurements of a clutter without moving targets.

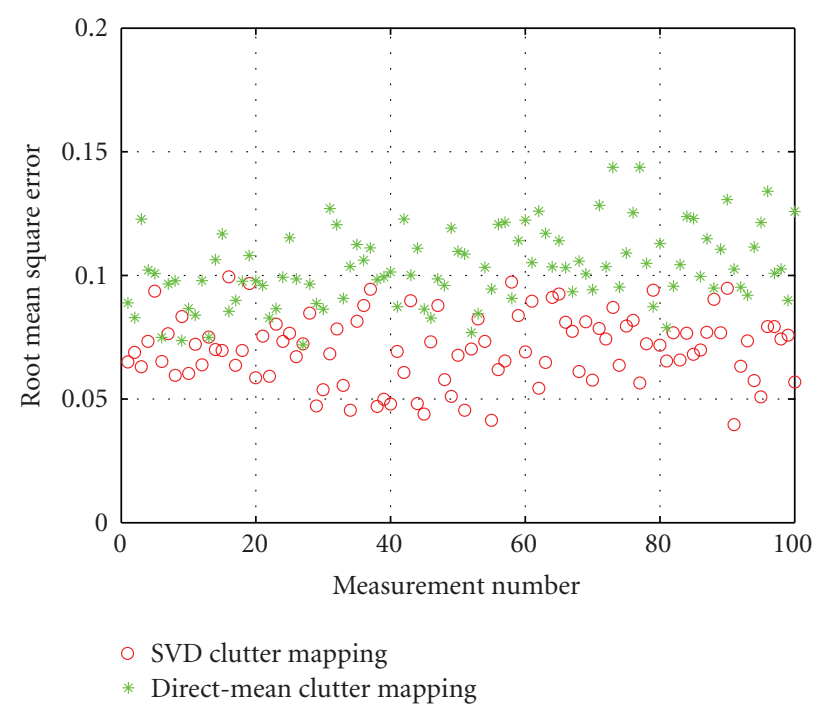

FIGURE 11: RMSE values for evaluation of the SVD and the directmean clutter mappings with a moving target.

moving object than manually measuring a static object by a ruler.

A clutter, an indoor environment without moving targets, is measured first. The measurement matrix $\mathbf{X}^{0}$ can be expressed by

$$
\mathbf{X}^{0}=\left[\mathbf{r}^{-99}, \mathbf{r}^{-98}, \ldots, \mathbf{r}^{-1}, \mathbf{r}^{0}\right]
$$

where we use $n=100$ measurements for $\mathbf{X}^{0}$, and the superscript $i \leq 0$ represents the clutter without targets. Using the SVD clutter mapping, the clutter map $\widehat{\mathbf{r}}_{e}^{0}$ can be obtained.

Then, a metal ball with a diameter of $20 \mathrm{~mm}$ is placed into the environment at 50 different locations statically. At each location, one measurement is performed, referred to as $\mathbf{r}^{i}, i=1,2, \ldots, 50$. 


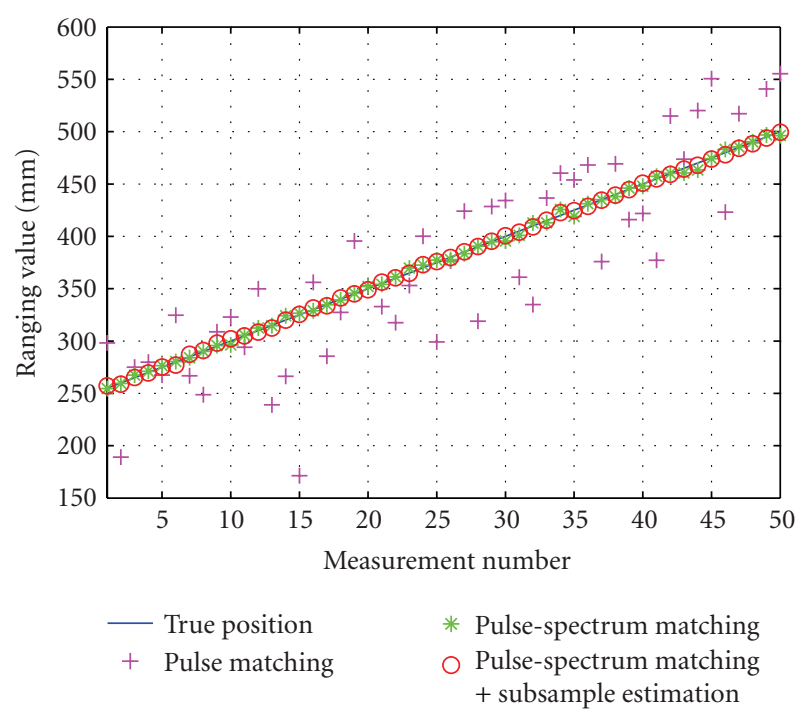

FIGURE 12: Evaluation of the ranging algorithms with the clutter map removal.

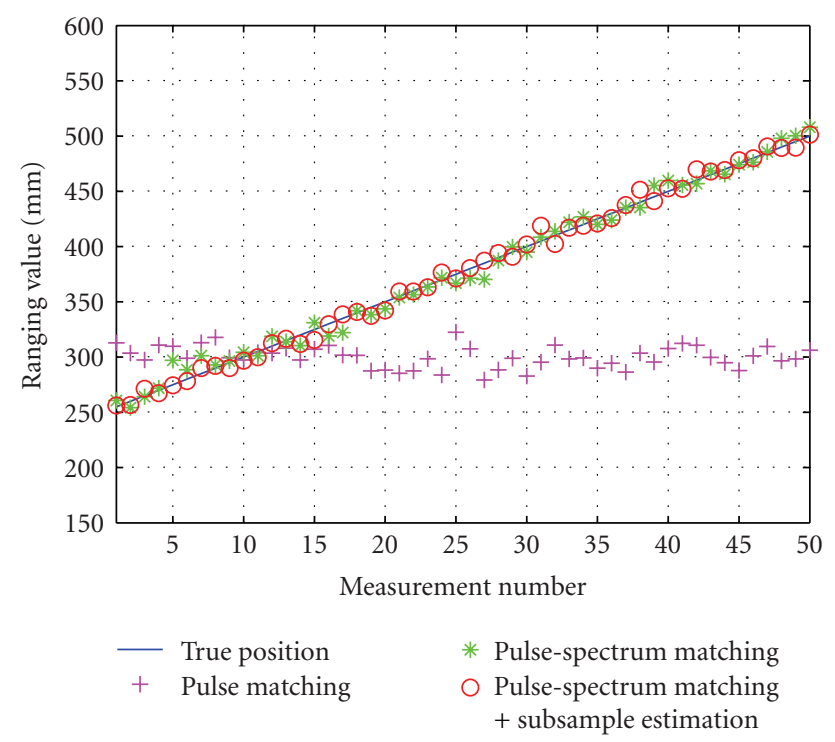

FIGURE 13: Evaluation of the ranging algorithms without the clutter map removal.

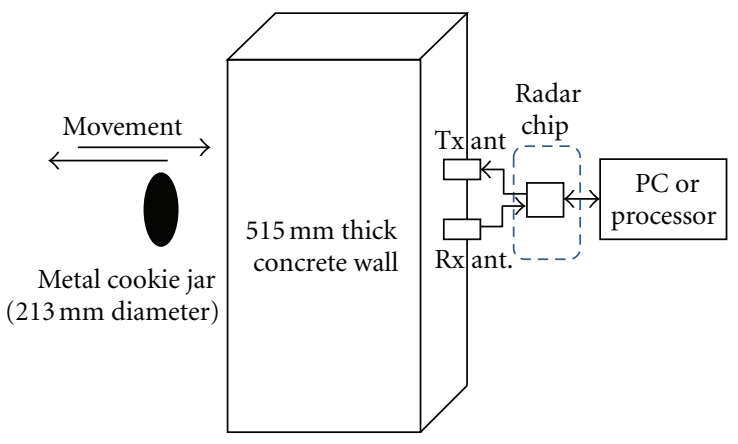

FIgURE 14: The setup for the evaluation of the one-dimensional tracking algorithm through a concrete corridor wall.

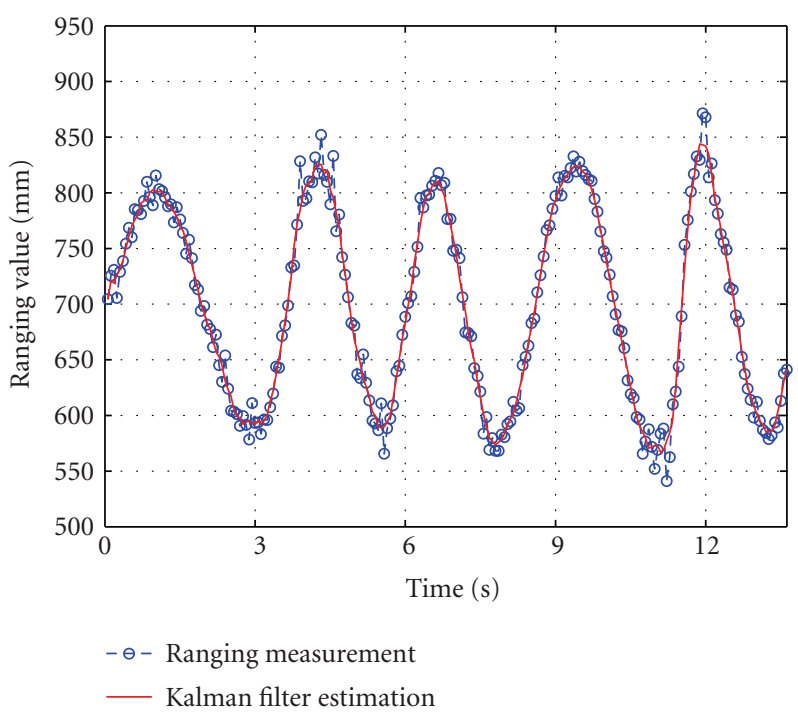

FIGURE 15: The estimated position by the Kalman filter tracking algorithm for the one-dimensional see-through-wall evaluation.

The emulating measurement matrix $\mathbf{X}^{i}$ for the "moving" metal ball is created off line by

$$
\mathbf{X}^{i}=\left[\mathbf{r}^{-99+i}, \mathbf{r}^{-98+i}, \ldots, \mathbf{r}^{-1+i}, \mathbf{r}^{i}\right]
$$

where $i=1,2, \ldots, 50$. For each $\mathbf{X}^{i}$, the adaptive clutter map $\widehat{\mathbf{r}}_{e}^{i}$ can be obtained, and the target signal $\widehat{\mathbf{r}}_{t}^{i}$ is acquired by the clutter map removal shown in (11). The ranging algorithms are then applied to the target signal $\widehat{\mathbf{r}}_{t}^{i}$ to have the ranging values for these 50 locations.

Figure 12 shows the ranging results over the 50 locations. As a comparison, the ranging without the clutter map removal is presented in Figure 13. Table 1 summarizes the RMSEs for the different ranging algorithms over the 50 locations. The true ranging values of the target are measured by a ruler with $1 \mathrm{~mm}$ resolution.

The ranging evaluation starts from $250 \mathrm{~mm}$, which is longer than the distance of $200 \mathrm{~mm}$ between the Tx and the $\mathrm{Rx}$ antennas, as explained in Section 2.2.

From Figure 12 and Table 1, it can be observed that the pulse-spectrum signature matching together with the subsample delay estimation gives the best result, achieving a resolution of $1.4 \mathrm{~mm}$, while the pulse signature matching provides much less accurate ranging. Furthermore, it can be concluded that the clutter map removal is very critical for the ranging accuracy.

The pulse-spectrum signature matching is a fast algorithm, since it involves only linear transformations (STFT) and calculations of cross-correlation. This makes the algorithm a very useful one in real-time applications, such as tracking.

8.3. Tracking. It is difficult for us to evaluate the tracking algorithm quantitatively, since the off-line method is not valid for real-time applications, and we do not have other means to get the accurate values of the position and the 
TABLE 1: Comparison of the ranging techniques in terms of RMSE between the real and estimated position for each measurement $i$.

\begin{tabular}{lcc}
\hline Algorithm & $\begin{array}{c}\text { Without clutter } \\
\text { map removal }\end{array}$ & $\begin{array}{c}\text { With clutter map } \\
\text { removal }\end{array}$ \\
\hline $\begin{array}{l}\text { Pulse signature matching } \\
\text { Pulse-spectrum signature }\end{array}$ & $75.4(\mathrm{~mm})$ & $48.1(\mathrm{~mm})$ \\
$\begin{array}{l}\text { matching } \\
\begin{array}{l}\text { Pulse-spectrum signature } \\
\text { matching + subsample } \\
\text { delay estimation }\end{array}\end{array}$ & $5.0(\mathrm{~mm})$ & $2.6(\mathrm{~mm})$ \\
\hline
\end{tabular}

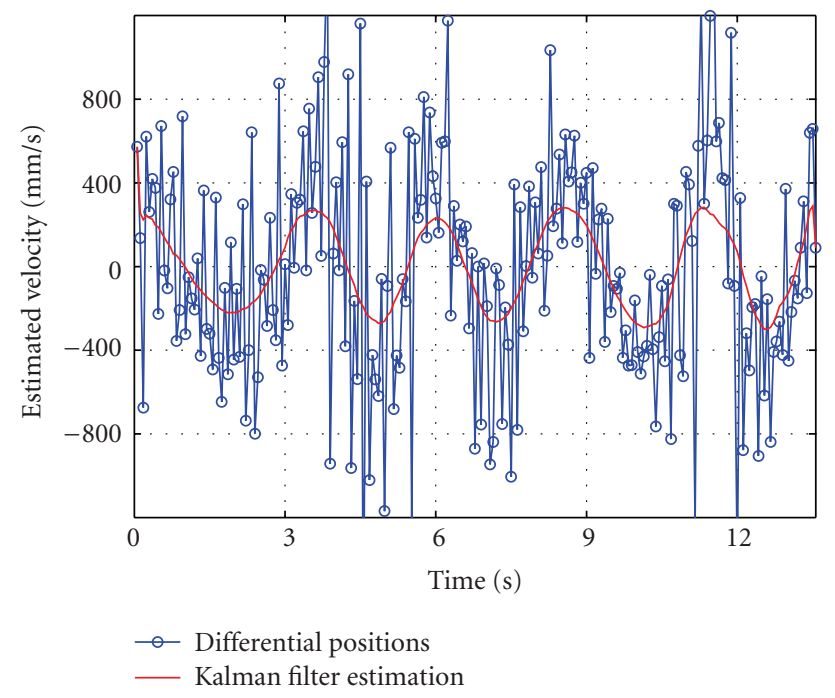

FIgURE 16: The estimated velocity by the Kalman filter tracking algorithm for the one-dimensional see-through-wall evaluation.

velocity of a moving target. The qualitative evaluation is accordingly applied.

Figure 14 shows the setup for one-dimensional tracking evaluation, by using the UWB radar as a see-through-wall radar. The Tx and the Rx SGBT antennas are located on one side of a $515 \mathrm{~mm}$ thick concrete corridor wall. The target, a $213 \mathrm{~mm}$-in-diameter metal cookie jar, is moved back and forth manually behind and orthogonally to the wall.

Figures 15 and 16 show the estimated positions and velocities by using the tracking algorithm. It can be seen that the Kalman filter algorithm gives a very reasonable result: the moving target has slightly different period and amplitude of the back and forth "oscillation" of both the position and the velocity, which agrees well with the movement by a human hand.

It should be noted that if the target is not moving orthogonally to the wall, the one-dimensional tracking algorithm with one radar device can only discriminate the distance between the target and the antennas, but not the positions.

The two-dimensional tracking algorithm is evaluated also qualitatively. Figure 17 (left) shows the setup: two UWB radars are located orthogonally, and a metal object is moved manually. The graphic user interface implemented in Matlab shows the target with a red dot. We have observed that the red dot follows the movement of the target very well. An image

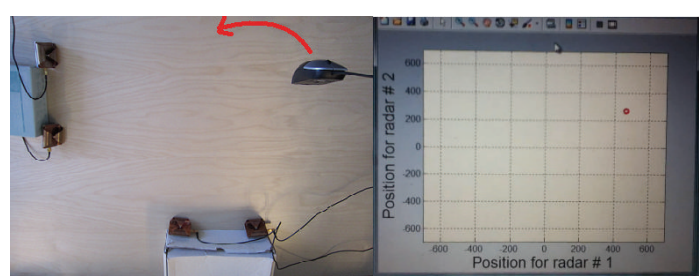

FIGURE 17: An orthogonal measurement setup for two-dimensional tracking (left) and the graphical user interface implemented in Matlab (right), where the red dot indicates the location of the target.

printed from the screen is shown in Figure 17 (right) for the illustration purpose.

\section{Conclusion}

A compact UWB radar system for indoor and throughwall ranging and tracking of moving objects has been built up by using the compact self-grounded Bow-Tie antennas and the low-cost Novelda transceiver. Robust and accurate algorithms for ranging and tracking have been developed. The evaluation by measurements shows that the ranging resolution of this UWB system has achieved to $1.4 \mathrm{~mm}$ RMS accuracy, and a fast and real-time tracking solution for through wall is obtained.

The main contribution of this UWB radar system is that the system itself has commercially low-cost, compact size. Equipped with the ranging algorithms, it provides a very high time domain resolution. Furthermore, the penetration ability together with the high resolution and the compact system size gives possibilities for many applications. However, the trade-off here is that due to the small size of the system, the dynamic range is relatively low, and therefore, it can only be used for short-range applications.

\section{Acknowledgments}

This work is a collaboration between Imego AB (The Institute of Micro and Nanotechnology in Gothenburg) and Chalmers University of Technology. The authors would like to thank Kenneth Kalmstrom, Lars Landen, Peter Bjorkholm, Jakob Blomgren, Dimitar Kolev, and Sohaib Maalik for their help in the project. This work has been supported in part by The Swedish Foundation for Strategic Research (SSF) within the Strategic Research Center Charmant.

\section{References}

[1] M. Z. Win, D. Dardari, A. F. Molisch, W. Wiesbeck, and J. Zhang, "History and applications of UWB," Proceedings of the IEEE, vol. 97, no. 2, pp. 198-204, 2009.

[2] P. J. Hall, "The square kilometre array: an international engineering perspective," Experimental Astronomy, vol. 17, no. 1-3, pp. 5-16, 2004.

[3] Q. Ren, "Energy detection performance analysis for UWB radar sensor networks," Eurasip Journal on Wireless Communications and Networking, vol. 2010, Article ID 709723, 2010. 
[4] J. Taylor, Ultra-Wideband Radar Technology, CRC, 2001.

[5] R. J. Fontana, "Recent system applications of short-pulse ultra-wideband (UWB) technology," IEEE Transactions on Microwave Theory and Techniques, vol. 52, no. 9, pp. 2087-2104, 2004.

[6] Novelda, http://www.novelda.no/.

[7] J. Yang and A. Kishk, "A novel low-profile compact directional ultra-wideband antenna: the self-grounded Bow-Tie antenna," IEEE Transactions on Antennas and Propagation, vol. 60, no. 3, pp. 1214-1220, 2012.

[8] J. Yang and A. Kishk, "The self-grounded Bow-Tie antenna," in Proceedings of the IEEE AP-S International Symposium on Antennas Propagation, Spokane, Washington, July 2011.

[9] J. Yang, X. Chen, N. Wadefalk, and P. S. Kildal, "Design and realization of a linearly polarized eleven feed for $1-10 \mathrm{GHz}$," IEEE Antennas and Wireless Propagation Letters, vol. 8, pp. 6468, 2009.

[10] J. Yang, M. Pantaleev, P. S. Kildal et al., "Cryogenic 2-13 GHz eleven feed for reflector antennas in future wideband radio telescopes," IEEE Transactions on Antennas and Propagation, vol. 59, no. 6, pp. 1918-1934, 2011.

[11] X. Zhang, X. Yan, J. Liu, J. Yang, and J. Morris, "Design of printed monopole antennas on liquid crystal polymer substrates," Journal of Infrared, Millimeter, and Terahertz Waves, vol. 31, no. 4, pp. 469-480, 2010.

[12] J. Yang, M. Pantaleev, P.-S. Kildal, and L. Helldner, "Design of compact dual-polarized 1.2-10 GHz Eleven feed for decade bandwidth radio telescopes," IEEE Transactions on Antennas and Propagation, vol. 60, no. 5, pp. 2210-2218, 2012.

[13] A. Razavi and J. Yang, "Investigation of penetration ability of UWB antennas in nearfield sensing applications," in Proceedings of the 6th European Conference on Antennas and Propagation (Eu-CAP '12), IEEE, Prague, Czech Republic, March 2012.

[14] Y. Yu, S. Maalik, J. Yang et al., "A new UWB radar system using UWB CMOS chip," in Proceedings of the 5th European Conference on Antennas and Propagation (EUCAP '11), pp. 771-775, April 2011.

[15] W. Wiesbeck, G. Adamiuk, and C. Sturm, "Basic properties and design principles of UWB antennas," Proceedings of the IEEE, vol. 97, no. 2, pp. 372-385, 2009.

[16] A. Laub, Matrix Analysis for Scientists and Engineers, Society for Industrial Mathematics, 2005.

[17] P. Woodward, Probability and Information Theory with Applications to Radar, Artech House, 1980.

[18] G. Turin, "An introduction to matched filters" IRE Transactions on Information Theory, vol. 6, no. 3, pp. 311-329, 1960.

[19] A. Farina, "Introduction to radar signal and data processing: the opportunity," Tech. Rep. DTIC Document, 2006.

[20] E. Jacobsen and R. Lyons, "The sliding DFT," IEEE Signal Processing Magazine, vol. 20, no. 2, pp. 74-80, 2003.

[21] Y. Jin and Z. Hao, "Gaussian window of optimal timefrequency resolution in numerical implementation of shorttime Fourier transform," Applied Mechanics and Materials, vol. 48-49, pp. 555-560, 2011.

[22] G. Strang, Introduction to Linear Algebra, Wellesley Cambridge, 4th edition, 2009.

[23] K. R. Rao and P. C. Yip, The Transform and Data Compression Handbook, Chapter 2, CRC, 2001.

[24] R. Bracewell, The Fourier Transform and Its Applications, McGraw-Hill, Boston, Mass, USA, 3rd edition, 2000.
[25] R. Brown and P. Hwang, Introduction to Random Signals and Applied Kalman Filtering, vol. 1, John Wiley \& Sons, New York, NY, USA, 1992.

[26] S. Kay, Fundamentals of Statistical Signal Processing: Estimation Theory, Prentice-Hall, 1993. 

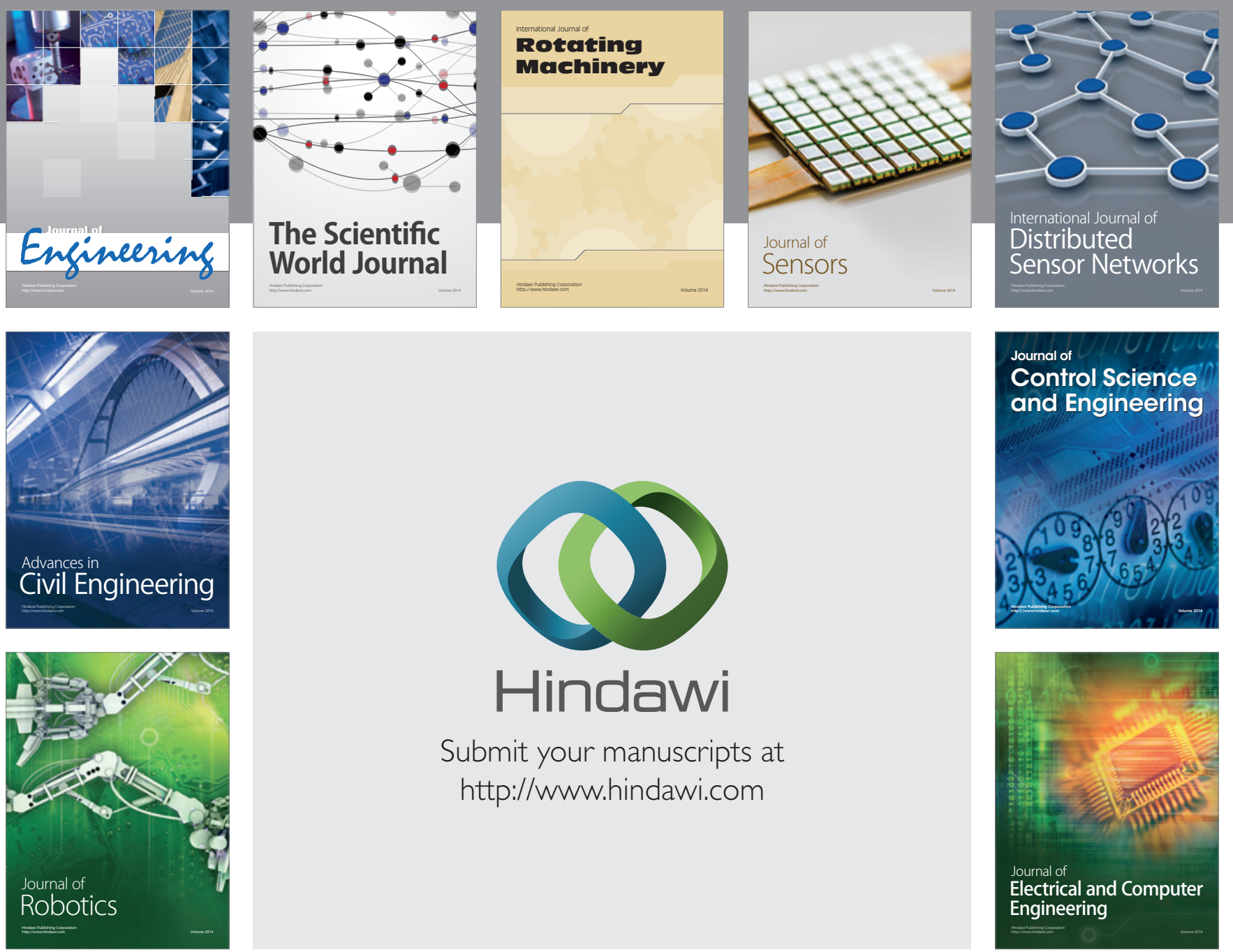

Submit your manuscripts at

http://www.hindawi.com
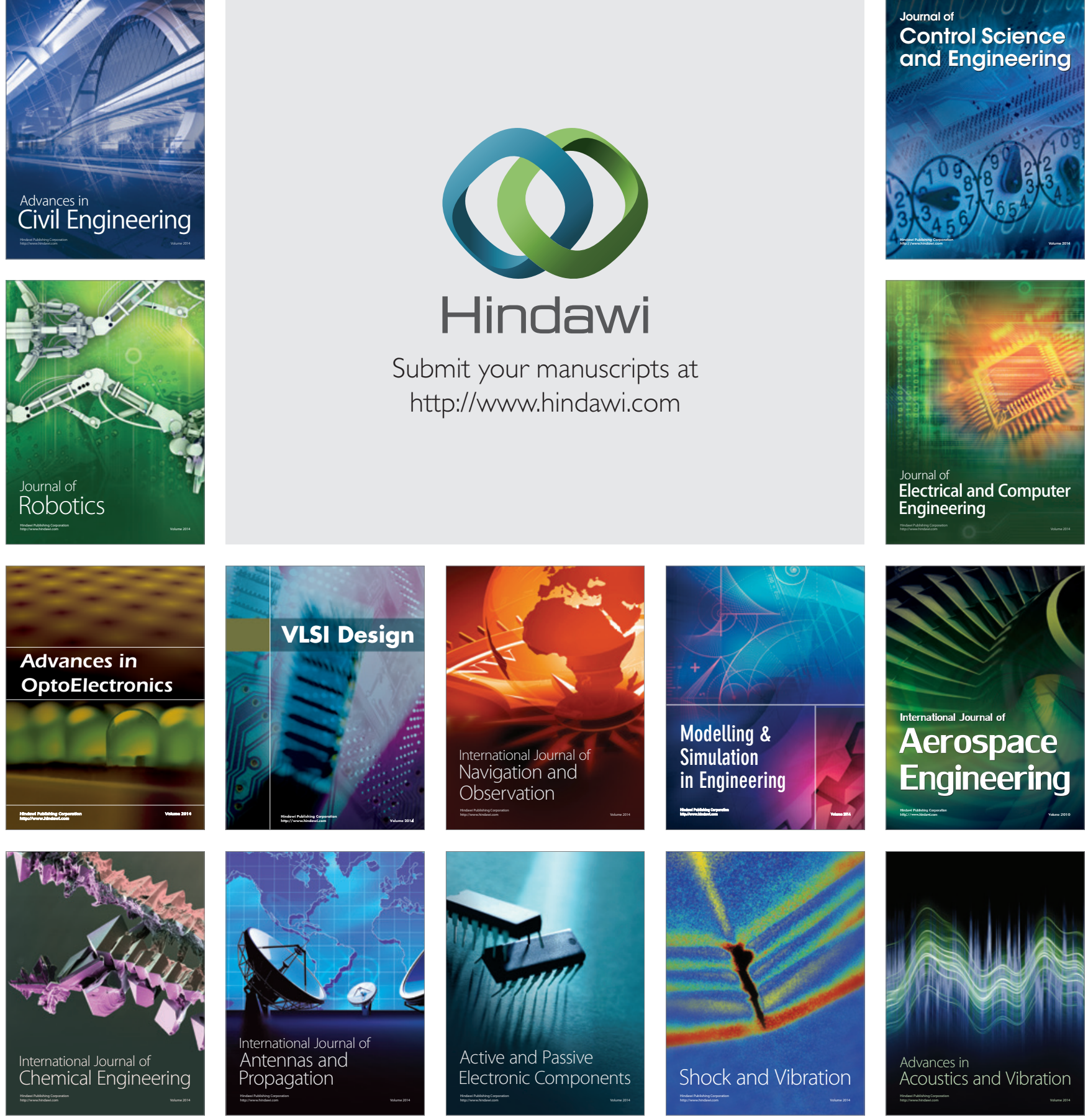